\title{
On Monochromatic Arithmetic Progressions Having Odd Step
}

Veselin Jungic

\section{CONTENTS}

1. Introduction

2. The Experiment

3. The Program and the Results

4. A Further Step

Acknowledgements

References
We perform an experiment concerning certain sets of 2-colorings and the existence of monochromatic arithmetic progressions of odd step. We use the results of the experiment to prove more general statements.

\section{INTRODUCTION}

The questions we address are of the following type: Is it possible to state a density characterization of a certain set of finite colorings?

Let $\mathbb{N}$ be the set of positive integers, let $\mathbb{N}_{0}=$ $\mathbb{N} \cup\{0\}$, and let [,] form closed intervals in $\mathbb{N}_{0}$. For $r \in \mathbb{N}$, an $r$-coloring (of $\mathbb{N}$ ) is a map $f: \mathbb{N} \rightarrow A$ with $|A|=r$. A coloring is an $r$-coloring for some $r$. If $f$ is an $r$-coloring and if $B \subseteq \mathbb{N}$ satisfies $|f(B)|=1$, we say that $B$ is $f$-monochromatic. An arithmetic progression of length $k$ and step $d$, where $k, d \in \mathbb{N}$ and $k>1$, is a set of the form

$$
\{a+(i-1) d: i \in[1, k]\} \quad \text { for some } a \in \mathbb{N} .
$$

Van der Waerden's theorem [1927] on arithmetic progressions says that for any coloring $f$ and any $k \in \mathbb{N}$ there is an $f$-monochromatic arithmetic progression of length $k$.

Szemerédi's theorem [1975] gives a criterion on where to look for long arithmetic progressions: If a set $S \subseteq \mathbb{N}$ has positive upper density, that is, if

$$
\bar{d}(S):=\limsup _{N \rightarrow \infty} \frac{|S \cap[1, N]|}{N}>0,
$$

then $S$ contains arbitrarily long arithmetic progressions.

Brown, Graham, and Landman [Brown et al. 1999] study subsets $L$ of $\mathbb{N}$ such that van der Waerden's theorem can be strengthened to guarantee the existence of arbitrarily long $f$-monochromatic progressions having step in $L$. 
For $r \in \mathbb{N}$ we say that a subset $L$ of $\mathbb{N}$ is $r$-large if every $r$-coloring yields arbitrarily long monochromatic progressions having step in $L$. We say that $L$ is large if it is $r$-large for every $r$. Perhaps surprisingly, there are many large sets; for example, for any $m \in \mathbb{N}$, the set $m \mathbb{N}$ is large.

Brown, Graham, and Landman have conjectured that any 2-large set is large.

If $p \in \mathbb{C}[x]$ satisfies $p(\mathbb{Z}) \subseteq \mathbb{Z}$ and $p(0)=0$, the set

$$
|p(\mathbb{Z})|:=\{|p(n)|: n \in \mathbb{Z}\}
$$

is large [Brown et al. 1999; Bergelson and Leibman 1996]. This second reference proves even more. Here we give a very special case of [Bergelson and Leibman 1996, Theorem $B_{0}$ ].

If $S \subseteq \mathbb{N}$ has positive upper density and if a polynomial $p \in \mathbb{C}[x]$ satisfies $p(\mathbb{Z}) \subseteq \mathbb{Z}$ and $p(0)=0$, then $S$ contains arbitrarily long monochromatic arithmetic progressions having step in $|p(\mathbb{Z})|$.

It is not known whether for any large set $L$ and any set $S$ of positive upper density, $S$ contains arbitrarily long monochromatic arithmetic progressions having step in $L$.

On the other hand, the set of all odd positive numbers is not 2-large. For example, let $n \in \mathbb{N}$ and consider the 2-coloring $g_{n}: \mathbb{N} \rightarrow\{0,1\}$ given by

$$
g_{n}(i)=1 \Longleftrightarrow 2^{n} \mid i
$$

Clearly, an arithmetic progression of odd step and length $2^{n}$ cannot be $g_{n}$-monochromatic. At the same time, the set $S=\left\{i: 2^{n} \nmid i\right\}$ satisfies

$$
\frac{\left|S \cap\left[1,2^{n} k\right]\right|}{2^{n} k}=1-\frac{1}{2^{n}} .
$$

Thus, for any $\varepsilon>0$ there is a 2-coloring of $\mathbb{N}$ such that the density of elements in one color is greater than $1-\varepsilon$, but there are no long arithmetic progressions of odd step in that color.

Another example of a 2-coloring that avoids long monochromatic arithmetic progressions having odd step is the following. Fix an even positive integer $n=2 p$, and define $f_{n}: \mathbb{N} \rightarrow\{0,1\}$ by alternating chunks of length $n-1$ :

$$
\begin{aligned}
f_{n}(i) & =0 \Longleftrightarrow \\
i & \in[2 t(n-1)+1,(2 t+1)(n-1)] \text { for some } t \in \mathbb{N}_{0} .
\end{aligned}
$$

Now regard $f_{2 p}$ as an array $A_{p}=\left\{a_{i, j}\right\}$ with $2 p$ columns and infinitely many rows, read row by row:

$$
a_{i, j}=f_{2 p}(2 i p+j) \quad \text { for } i \in \mathbb{N}_{0} \text { and } j \in[1,2 p] .
$$

For example, $A_{3}$ is the array that starts with

$\begin{array}{llllll}0 & 0 & 0 & 0 & 0 & 1 \\ 1 & 1 & 1 & 1 & 0 & 0 \\ 0 & 0 & 0 & 1 & 1 & 1 \\ 1 & 1 & 0 & 0 & 0 & 0 \\ 0 & 1 & 1 & 1 & 1 & 1\end{array}$

and continues downward with identical blocks. The density of 0 's in any odd column of $A_{p}$ is $p /(2 p-1)$, and that of 1 's is $(p-1) /(2 p-1)$.

As shown in [Jungic $\geq 2000$ ], not only does $f_{2 p}$ avoid $2 p$-term monochromatic arithmetic progressions of odd step, but the same is true of any 2coloring obtained by changing arbitrarily many 1's to 0 's in $A_{p}$, provided the changes occur only on odd columns and one odd column remains unchanged. The same is true if we change some 0's to 1 's in $A_{p}$, confining the changes to even columns and leaving one even column unchanged.

Now let $f:[1,2 p(2 p-1)] \rightarrow\{0,1\}$ be represented by an array $A$ obtained from the first $2 p-1$ rows of $A_{p}$ by changing some 1's to 0's on even columns. One can show that if the number of 0 's on any even column of $A$ is at least $2 p-3$, there is a $2 p$-term $f$ monochromatic arithmetic progression of odd step. (The number of 0's on any odd column stays at $p$.)

These observations lead to the following question.

Question. Is there $t \in(0,1)$ such that if a 2-coloring is represented by a $2 p$-column array each of whose columns has upper density of 0's greater than $t$, then there must be an arithmetic progression of odd step and length $2 p$ all of whose elements have color 0 ?

An affirmative answer to this question could be an important step towards the resolution of the conjecture that every $r$-large set is large.

Section 2 describes an experiment related to this question. Section 3 describes the program used to run the experiment, and gives the results of the experiment. These results suggest that the answer to the question is affirmative in the case $p=2$ and that a possible value for $t$ is $\frac{1}{2}$. In Section 4 we prove certain statements that are both inspired and justified by our experiment. 


\section{THE EXPERIMENT}

Let $p=2$. The density of 0 's on odd columns of $A_{2}$ equals $\frac{2}{3}$ and the density of 0 's on even columns of $A_{2}$ equals $\frac{1}{3}$. It is easy to see that if we change any 0 that belongs to an even column, the new array represents a coloring that admits a 4-term monochromatic arithmetic progression of step 1 .

For convenience we will think of our 2-colorings as being black and white. We would like to get some idea of what happens with 4-term monochromatic arithmetic progressions with odd step if a given 2coloring is such that in every mod 4 congruence class the density of black elements is $\frac{1}{2}$.

Let $n \in \mathbb{N}$. We will consider 2 -colorings of $[1,8 n]$ with the property that each mod 4 class has exactly $n$ black elements. As a source of 2-colorings where each mod 4 class has upper density of black elements equal to $\frac{1}{2}$, we will consider a subdivision $a_{0}=0<$ $a_{1}<\cdots<a_{m}=n$ of the interval $[0, n]$, so that

$$
[1,8 n]=\bigcup_{i=0}^{m-1}\left[8 a_{i}+1,8 a_{i+1}\right],
$$

and 2-colorings $\chi:[1,8 n] \rightarrow\{\circ, \bullet\}$ such that, within each interval $\left[8 a_{i}+1,8 a_{i+1}\right]$, the density of black elements in each mod 4 class is $\frac{1}{2}$. Such a 2-coloring $\chi$ is said to be compatible with the subdivision $\left\{a_{i}\right\}_{i=0}^{m}$. The number of 2-colorings compatible with $\left\{a_{i}\right\}_{i=0}^{m}$ is

$$
\prod_{i=0}^{m-1}\left(\begin{array}{c}
2\left(a_{i+1}-a_{i}\right) \\
a_{i+1}-a_{i}
\end{array}\right)^{4}
$$

Experiment. For given $k, l \in \mathbb{N}$, set $n=k l$, and consider the set of 2-colorings $\chi:[1,8 n] \rightarrow\{\circ, \bullet\}$ that are compatible with the subdivision $\{2 k i\}_{i=0}^{l}$ of $[0, n]$. Find the fraction of such 2-colorings that avoid monochromatic arithmetic progressions with four terms and odd step.

\section{THE PROGRAM AND THE RESULTS}

We ran this experiment for $(k, l) \in[1,3] \times[1,5]$, using a program written in $\mathrm{C}$ and based on the following steps.

1. Fix $k$ and $l$.

2. Form the set $C_{k}$ of all $2 k$-tuples with $k$ white and $k$ black dots.

3. Form the set $D_{k}$ of all $8 k$-tuples obtained by interleaving 4 elements of $C_{k}$ (so each mod 4 class of an element of $D_{k}$ is in $C_{k}$ ).

4. Find the subset $\bar{D}_{k} \subseteq D_{k}$ consisting of $8 k$-tuples that avoid monochromatic progressions of odd step and length 4 . This is done by examining each possible step size and starting point of a progression.

5. Form the set $E_{k, l}$ of all $8 k l$-tuples obtained by concatenating $l$ elements of $\bar{D}_{k}$.

6. Count the elements of $E_{k, l}$ that avoid monochromatic arithmetic progressions of odd step and length 4.

Two key factors make this idea efficient. First, $\bar{D}_{k}$ can be easily stored in memory, being a relatively small set:

\begin{tabular}{|rrl|}
\hline$k$ & $\left|\bar{D}_{k}\right|$ & $\left|\bar{D}_{k}\right| /\left|D_{k}\right|$ \\
\hline 1 & 11 & 1 in 1.45 \\
2 & 329 & 1 in 25.18 \\
3 & 6354 & 1 in 3.94 \\
\hline
\end{tabular}

Second, for any element of $\bar{E}_{k, l}$ the prefix of length $8 k(l-1)$ must be in $\bar{E}_{k,(l-1)}$; therefore the search space in Step 6 is much reduced.

The results of the experiment appear in Table 1. They suggest that $t=\frac{1}{2}$ may possibly yield an affirmative answer to the Question of page 968 in the case $p=2$.

\begin{tabular}{|c|ccccc|}
\hline$k$ & \multicolumn{4}{|c|}{$\left|\bar{E}_{k, l}\right|$ for } & $l=4$ \\
& $l=1$ & $l=2$ & $l=3$ & $l=5$ \\
\hline 1 & $11=1$ in 1.45 & $54=1$ in 4.741 & $55=1$ in 74.473 & $21=1$ in 3120.762 & 0 \\
2 & $329=1$ in 3.94 & $4254=1$ in 394.83 & $866=1$ in 2513605 & $12=1$ in 235092492288 & 0 \\
3 & $6354=1$ in 25.180 & $38188=1$ in 670367 & $26=26$ in $20^{12}$ & 0 & 0 \\
\hline
\end{tabular}

TABLE 1. Among all 2-colorings $\chi$ of the type considered in the Experiment (see end of Section 2), we give the number of those that avoid monochromatic arithmetic progressions of length 4 and odd step, and show what fraction of the total they represent. 
For comparison, by [Jungic $\geq 2000$, Corollary 5] that there are at least $2\left(2^{12}-1\right) 2$-colorings of the interval $[1,120]$ that avoid 4 -term monochromatic arithmetic progressions of odd step.

The fact that the rightmost column of Table 1 is made up of zeros can be restated as follows:

Theorem 1. Let $k \in\{1,2,3\}$. Suppose a 2-coloring of $\mathbb{N}$ has in interval of length $40 k$ which, when regarded as a coloring of $[1,40 k]$, is compatible with the subdivision $\{8 i k\}_{i=0}^{5}$. Then there are $\chi$-monochromatic progressions of either color having 4 terms and odd step no greater than $13 k$.

\section{A FURTHER STEP}

This section presents a few statements inspired and justified by our experiment.

We define a subset $T \subset \mathbb{N}^{2}$ as follows. Fix $l, t \in \mathbb{N}$. For any $k_{1}, \ldots, k_{t} \in[1, l]$, we regard

$$
\left\{0, k_{1}, k_{1}+k_{2}, \ldots, K:=k_{1}+k_{2}+\cdots+k_{t}\right\}
$$

as a subdivision of $[0, K]$ and ask whether all 2colorings of $[0,8 K]$ that are compatible with this subdivision (see Section 2) admit a black 4-term progression of odd step. If the answer is yes for all $k_{1}, \ldots, k_{t} \in[1, l]$, we have $(l, t) \in T$ by definition. Thus, for example, $(1,5) \in T$, as can be seen from the entry $k=1, l=5$ in Table 1 .

Complementarily, if some 2-coloring compatible with some sequence of the type above avoids 4 -term black progressions of odd step, then $(l, t) \notin T$. Thus $(2,2) \notin T$, again by Table 1 .

Proposition 2. If $(l, t) \in T$ then $t>1$.

Proof. Let $l \in \mathbb{N}$ and form the $2 l \times 4$ array whose odd columns are black at the top and white at the bottom, and whose even columns are white at the top and black at the bottom, as illustrated here for $l=3$ :

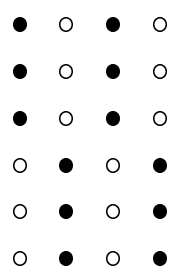

The corresponding coloring of $[1,8 l]$ is clearly compatible with $\{0, l\}$. A simple analysis shows that no black progression of odd step can have length 3 or more; therefore $(l, 1) \notin T$.

Lemma 3. Suppose $B$ and $A \subseteq B$ are sets whose cardinalities satisfy

$$
|A|>\left\lceil\left(1-\frac{1}{t}\right)|B|\right\rceil
$$

for some $t \in \mathbb{R}$. Then any partition of $B$ into sets of cardinality at most $t$ has at least one element that is a subset of $A$.

Proof. If not, we can take one element of $B \backslash A$ from each set in the partition. This yields a set disjoint from $A$ having at least $[|B| / t\rceil$ elements, in contradiction with $(4-1)$.

Theorem 4. Let $n \in 8 \mathbb{N}$ and let a subdivision $\left\{a_{i}\right\}_{i=0}^{m}$ of the interval $\left[1, \frac{n}{8}\right]$ be given. If there is $(l, t) \in T$ such that

$$
m>\max \left\{n z, \frac{8 l t z}{8(l+t) z-t}\right\}
$$

where

$$
z=\max \left\{\frac{1}{8 l}, \frac{t}{8(l+t)}\right\},
$$

then for any 2 -coloring $\chi$ of the interval $[1, n]$ compatible with $\left\{a_{i}\right\}_{i=0}^{m}$ there are 4-term $\chi$-monochromatic arithmetic progressions of odd step in both colors.

Proof. Let $I=\left\{i: a_{i+1}-a_{i} \leq l\right\}$ and $k=|I|$. Then

$$
\begin{aligned}
n & =8\left(\sum_{i \in I}\left(a_{i+1}-a_{i}\right)+\sum_{i \notin I}\left(a_{i+1}-a_{i}\right)\right) \\
& \geq 8((l+1) m-l k) .
\end{aligned}
$$

Thus,

$$
k \geq \frac{l+1}{l} m-\frac{n}{8 l}>\frac{8(l+1) z-1}{8 l z} m .
$$

From the assumption

$$
m>\frac{8 l t z}{8(l+t) z-t}
$$

we conclude that

$$
\frac{1}{m}<\frac{1}{t}+\frac{1}{l}-\frac{1}{8 l z}=\frac{8(l+1) z-1}{8 l z}-\frac{t-1}{t} .
$$

It follows that there is $p \in \mathbb{N}$ such that

$$
\frac{t-1}{t}<\frac{p}{m}<\frac{8(l+1) z-1}{8 l z}<\frac{k}{m}
$$


thus

$$
k>\left\lceil\left(1-\frac{1}{t}\right) m\right\rceil .
$$

Now we take $B=[1, t\lfloor m / t\rfloor]$ and $A=I \cap B$ in Lemma 4 , partitioning $B$ into $\lfloor m / t\rfloor$ intervals with $t$ elements. It is easy to check that $(4-1)$ is satisfied. The lemma therefore guarantees that $I$ contains an interval with $t$ elements.

Let $\chi$ be a 2 -coloring of the interval $[1, n]$ that is compatible with $\left\{a_{i}\right\}_{i=0}^{m}$. Since $(l, t) \in T$, the restriction of $\chi$ to $[(i-1) t+1, i t]$, for $t$ consecutive values of $i \in T$, allows 4 -term $\chi$-monochromatic arithmetic progressions in both colors having odd step.

Theorem 5. Let $n \in 8 \mathbb{N}$ and let a subdivision $\left\{a_{i}\right\}_{i=0}^{m}$ of the interval $\left[1, \frac{n}{8}\right]$ be given. If there is $(l, t) \in T$ such that

$$
(l+1) m-l\left\lceil\left(1-\frac{1}{t}\right) m\right\rceil>\frac{n}{8},
$$

then for any 2-coloring $\chi$ of the interval $[1, n]$ compatible with $\left\{a_{i}\right\}_{i=0}^{m}$ there are 4-term $\chi$-monochromatic arithmetic progressions in both colors having odd step.

Proof. Let $I=\left\{i: a_{i+1}-a_{i} \leq l\right\}$ and $k=|I|$. Once more we have

$$
k>\left\lceil\left(1-\frac{1}{t}\right) m\right\rceil,
$$

and we conclude exactly as in the proof of the preceding theorem.

Combining this with the fact that $(1,5) \in T$ we get:
Corollary 6. Let $m \in \mathbb{N}$ be given. For any $n \in 8 \mathbb{N}$ such that

$$
2 m-\left\lceil\frac{4}{5} m\right\rceil>\frac{1}{8} n \geq m
$$

and for any 2 -coloring $\chi$ of the interval $[1, n]$ that is compatible with a subdivision of $\left[1, \frac{n}{8}\right]$ into $m$ subintervals, there are 4-term $\chi$-monochromatic progressions of odd step in both colors.

\section{ACKNOWLEDGEMENTS}

This work was inspired by a talk given by Jonathan Borwein. Thanks to Greg Fee for his help with the initial C program, and to Silvio Levy for suggestions that led to significant improvements in this paper.

\section{REFERENCES}

[Bergelson and Leibman 1996] V. Bergelson and A. Leibman, "Polynomial extensions of van der Waerden's and Szemerédi's theorems", J. Amer. Math. Soc. 9:3 (1996), 725-753.

[Brown et al. 1999] T. C. Brown, R. L. Graham, and B. M. Landman, "On the set of common differences in van der Waerden's theorem on arithmetic progressions", Canad. Math. Bull. 42:1 (1999), 25-36.

[Jungic $\geq 2000]$ V. Jungic, "On a set of 2-colorings". Submitted for publication.

[Szemerédi 1975] E. Szemerédi, "On sets of integers containing no $k$ elements in arithmetic progression", Acta Arith. 27 (1975), 199-245.

[van der Waerden 1927] B. L. van der Waerden, "Beweis einer Baudetschen Vermutung", Nieuw Arch. Wisk. 15 (1927), 212-216.

Veselin Jungic, Department of Mathematics and Statistics, Simon Fraser University, Burnaby, B.C., Canada V5A1S6 (vjungic@sfu.ca) 